\title{
Robust Iris Recognition Baseline for the Grand Challenge
}
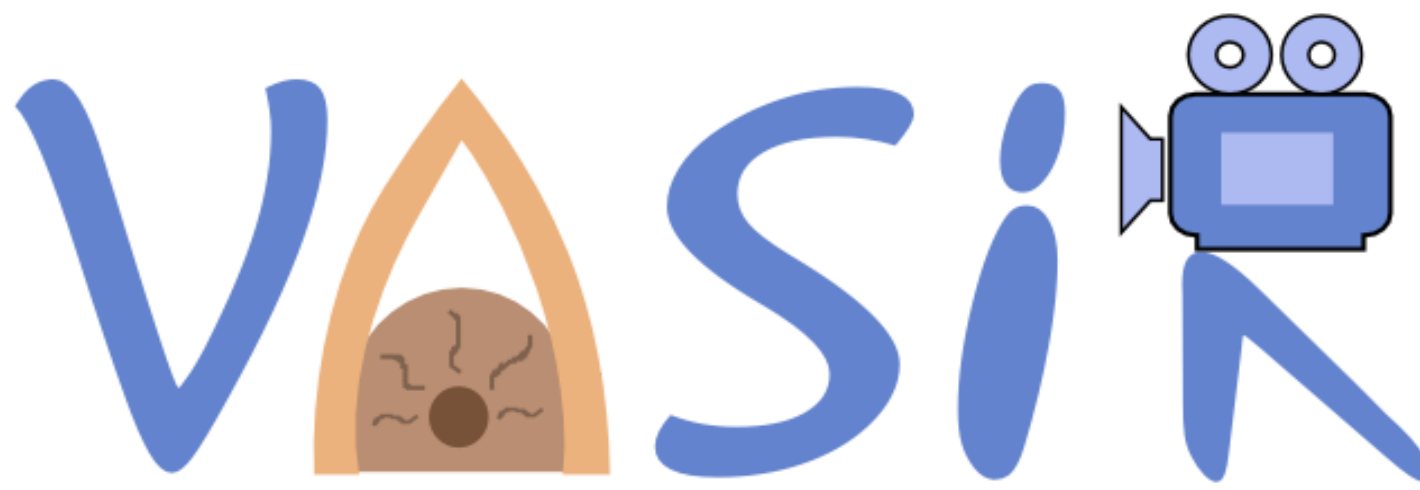

Video-based Automatic System for Iris Recognition

\author{
Yooyoung Lee \\ Ross J. Micheals \\ James J. Filliben \\ P. Jonathon Phillips
}

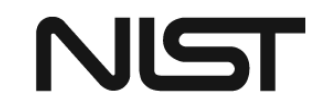

National Institute of Standards and Technology U.S. Department of Commerce 


\title{
Robust Iris Recognition Baseline for the Grand Challenge
}

\author{
Yooyoung Lee \\ Ross J. Micheals \\ P. Jonathon Phillips \\ Information Access Division \\ Information Technology Laboratory \\ James J. Filliben \\ Statistical Engineering Division \\ Information Technology Laboratory
}

National Institute of Standards and Technology Gaithersburg, MD 20899

March 2011

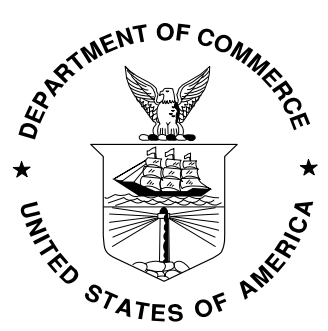

\section{U.S. Department of Commerce}

Gary Locke, Secretary

National Institute of Standards and Technology Patrick D. Gallagher, Director 


\begin{abstract}
Due to its distinctiveness, the human iris is a popular biometric feature used to identity a person with high accuracy. The "Grand Challenge" in iris recognition is to have an effective algorithm for subject verification or identification under a broad range of image and environmental conditions. This paper presents both baseline performance results and an enhanced version of VASIR (Video-based Automated System for Iris Recognition) as a response the challenge. We describe the details of the VASIR procedure and demonstrate its superiority over the IrisBEE algorithm. Finally, the image quality scores and how they relate to VASIR's performance are examined.
\end{abstract}

Keywords - biometrics, iris recognition, VASIR, baseline, benchmark, Hamming distance, image processing 


\section{Introduction}

Human physiological or behavioral characteristics can be integrated into a system to identify a per son; such a system is called a Biometric system. Specifically, the human iris is a highly distinctive fea ture that can provide a promising security application which we call iris recognition.

Current iris recognition, however, is often limited by significant input and environmental restrictions of the image acquisition. For example, an iris recognition system might perform well only for high quality images, which were captured with devices specially designed for the system. In the iris recognition community, the "Grand Challenge" is to create a robustly effective algorithm for subject identification that yields accurate results for a broad range of image and environmental conditions; e.g., a Near-Infrared (NIR) video-frame captured at a distance with an Iris On the Move (IOM) system which we call "distant-video-based" contrasted with a classical still-image acquisition system such as the LG 2200 EOU which we call "classical-still-based" iris recognition.

Video-based iris recognition at a distance is a relatively new research subject which still needs to overcome a number of issues. The main purpose of this paper is to provide a performance reference for video-based iris recognition, to motivate other researchers to promote their iris technology and to address current problems.

This paper also describes a particular iris recognition system VASIR (Video-based Automated System for Iris Recognition) which was developed to address such limitations that were shown in our existing works. A distinguishing part of VASIR is its ability to automatically detect the eye region and then to automatically select the best quality iris image from NIR face visible video captured at a distance. After such acquisition processing, VASIR carries out image comparison analysis to yield an individual verification [1].

To establish additional robustness in the VASIR system, two significant updates have been implemented:

1) Morphological preprocessing for improved accuracy of iris region segmentation, and

2) Modified Hamming distance (HD) matching methodology for improved false reject rates (FRR)

We also describe which performance metrics (e.g., false accept rate) are applied for the quantitativ e comparison of an algorithm's performance. In addition, this paper provides a detailed explanation of the test protocol that was utilized for our experiments.

To assess VASIR's performance, we address the following three specific questions:

1) Is VASIR robustly superior to IrisBEE?

2) Can VASIR's results be used as a state-of-the-art baseline for both classical-still-based and distant-video-based iris recognition?

3) How does VASIR performance change with the image quality?

The system was evaluated using ICE (Iris Challenge Evaluation) 2005 and MBGC (Multiple Biom etrics Grand Challenge) image datasets (publicly available) provided by the National Institute of Stan dards and Technology (NIST) [2][3].

Section 2 gives a brief overview of IrisBEE and VASIR. Section 2 describes the ICE and MBGC d atasets. Section 3 discusses a new approach of segmentation and matching methods. Section 5 describ es in detail VASIR's performance results vis-à-vis the three above-mentioned questions. 


\section{Overview of IrisBEE and VASIR}

Most iris-based systems currently in existence are for controlled classical-still-based iris recognition. IrisBEE, based on Masek's algorithm [2][4], was originally developed at NIST to be used as a reference implementation for such a classical-still-based iris recognition.

IrisBEE - as shown in Figure 1 - is comprised of five distinct steps for iris recognition. In the first step, an image of the subject's eye region is taken through classical image acquisition system (e.g., LG2200 EOU) - such an image is captured with careful image quality controls. The segmentation in the second step isolates the iris region from the rest of the acquired image. The isolated iris region is then normalized for comparison to another. Next, the features of the iris pattern are extracted from the normalized image and encoded to generate the iris template. Finally, the created iris template is compared with a template generated from an enrolled iris image.

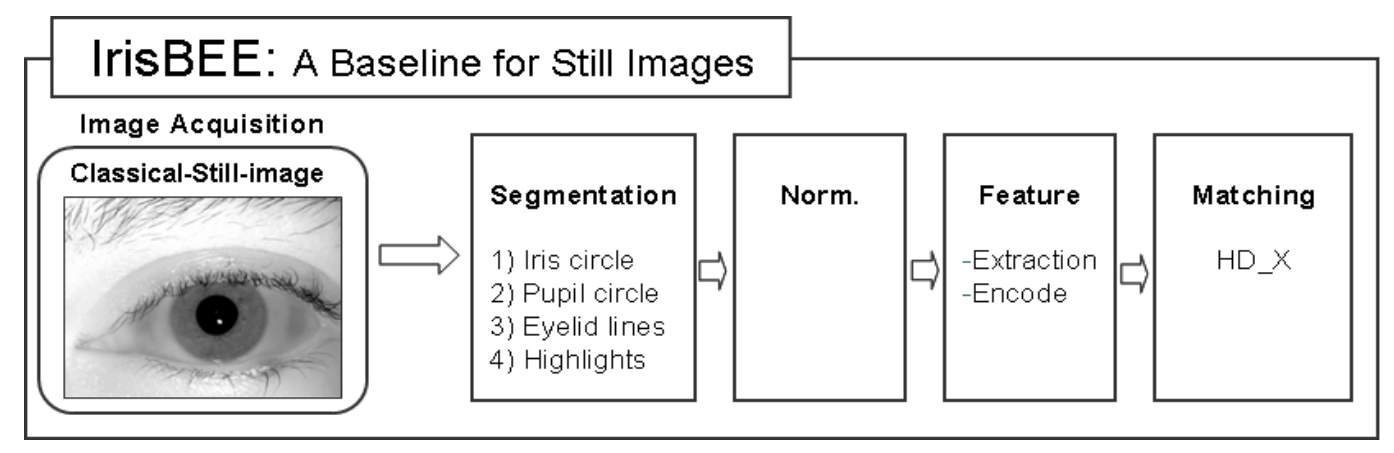

Figure 1 IrisBEE procedure

In contrast to the IrisBEE's algorithm, VASIR was developed with less constrained distant-videobased iris recognition in mind. VASIR also robustly allows performing classical-still to classical-still (SvsS), distant-video to classical-still (VvsS), and distant-video to distant-video (VvsV) iris recognition. In SvsS matching, a classical still-image is matched against other classical still-images of the same person that were captured by the same device (LG2200 EOU system). VvsS means that the video-frame captured at a distance (IOM system) is compared to classical still-images, captured by a different camera. In VvsV matching, the extracted iris region of distant-video frames is matched to other frames either from the same video or different video sequences of the same person.

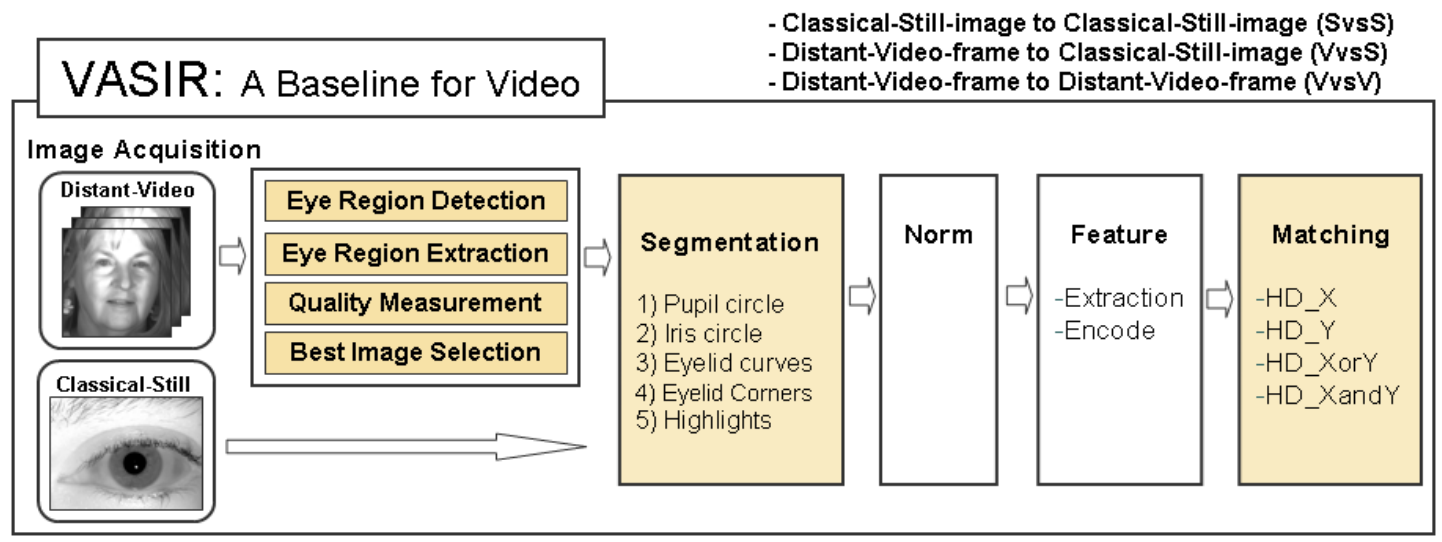

Figure 2 VASIR procedure (a case of video-distant vs classical-still) 
Figure 2 illustrates the procedural components of VASIR. The VASIR image acquisition step includes a facial-visible NIR video captured while a person walks through a portal, in addition to a classical still iris image. Four additional steps are used to identify a person from less constrained video at a distance. First, VASIR detects the eye region within each frame. Second, the system automatically extracts an eye region sub-image. Then the algorithm measures the quality of the detected eye region sub-images by calculating the edge density within the images. Finally, VASIR selects the best quality image out of all frames - a procedure we call Automated Image Quality Measurement (AIQM).

To facilitate comparing two iris images, Daugman's polar coordinate-based system [5] is then employed to normalize the iris region. The 1D-Log-Gabor Filter is used to process the feature extracting and feature encoding of the normalized iris images, as introduced by Yao et al [6]. VASIR was developed with the normalization and feature extraction based on Masek's algorithm [4].

\section{Datasets}

\section{A. Iris Challenge Evaluation (ICE) 2005}

The ICE 2005 data was collected at the University of Notre Dame in cooperation with NIST. The near-infrared iris still images are 640x480 in resolution and were captured by a LG EOU 2200 acquisition system.

Table 1 ICE2005 dataset (NIR_Iris_Still)

\begin{tabular}{|c|c|c|}
\hline Position & Num. of Images & Num. of Subjects \\
\hline Left Eye & 1,528 & 120 \\
\hline Right Eye & 1,425 & 124 \\
\hline
\end{tabular}

The total number of iris images is 2,953 and only 112 subjects exist for both right and left out of the total 132 subjects. In the original ICE2005 dataset, the image "246260.tiff" was mistakenly included in the left image set instead of in the right images, and consequently was set aside in our study [2].

\section{B. MBGC (Multiple Biometrics Grand Challenge)}

Our VASIR performance study used two different data types included in the MBGC dataset.

The near-infrared facial video (NIR_Face_Video or distant-video) were captured by an Iris On the Move (IOM) system; each frame is $2048 \times 2048$ pixels and the iris diameter is $\sim 100$ pixels. The nearinfrared iris still images (NIR_Iris_Still or classical-still) were collected by an LG2200 EOU and are $640 \times 480$ in resolution and the iris diameter is $\sim 200$ pixels [12].

Table 2 NIR_Face_Video captured by IOM system

\begin{tabular}{|c|c|c|}
\hline NIR_Face_Video & Version 1 & Version 2 \\
\hline Num. of the total video & 149 & 628 \\
\hline Num. of both eyes visible & 130 & 598 \\
\hline Total frames & 2,534 & 11,341 \\
\hline
\end{tabular}


Table 3 NIR_Iris_Still captured by LG2200 EOU system

\begin{tabular}{|c|c|c|}
\hline NIR_Iris_Still & Version 1 & Version 2 \\
\hline Left & 834 & 4,025 \\
\hline Right & 834 & 4,013 \\
\hline
\end{tabular}

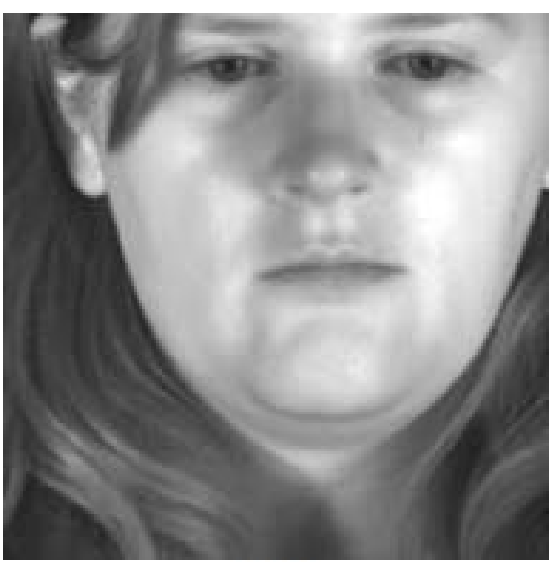

(a)

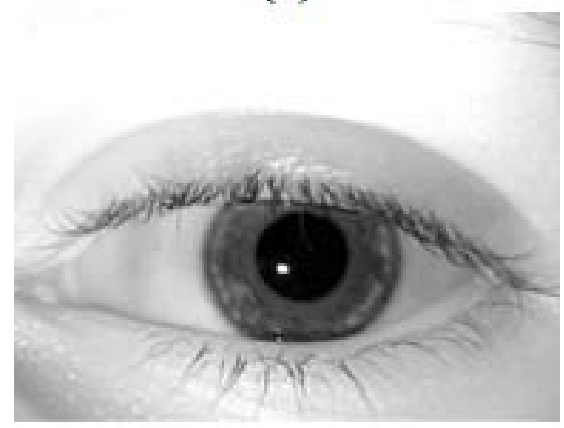

(c)

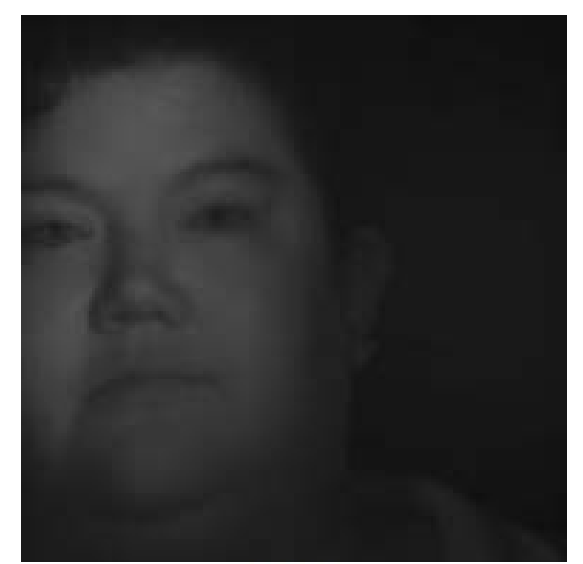

(b)

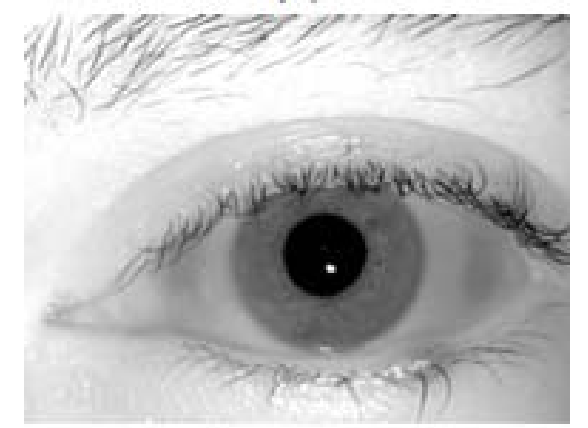

(d)

Figure 3 Illustration of differences between (a)-(b) NIR_Face_Video captured by a IOM and (c) - (d) NIR_Iris_Still captured by a LG2200 EOU;

(a) and (c) are from a person A, (b) and (d) are from a person

The detailed numbers for distant-video and classical-still iris images can be found in Table 2 and 3. MBGC has two versions of datasets. MBGCv2 is the larger dataset that are included the MBGCv1. Figure 3 shows the differences between NIR_Face_Video and NIR_Iris_Still in the MBGC dataset. 


\section{Segmentation and Matching}

\section{A. Segmentation}

In earlier version of VASIR, the segmentation components first detected the pupil boundary by fitting an ellipse to the contour points. In order to reduce noise in an image, a Gaussian filter was applied to the image before getting the binary threshold image [7]. Even though this led to better pupil segmentation results compared to IrisBEE, this was still not an optimal solution for reducing noise in images caused by blurriness, eyelashes, eyelid, glasses, lens distortion, reflections, or shadows.

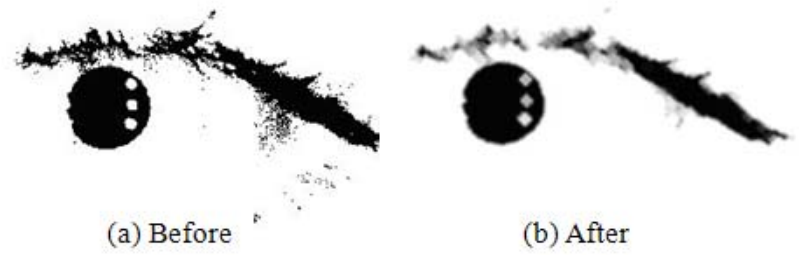

Figure 4 Example of noise reduction

VASIR now applies a Gaussian filter to the binary threshold image and uses morphological opening and closing later on. Opening of an image is an erosion followed by a dilation to remove small objects from a binary image while preserving the shape and size of larger objects within the image. Closing on the other hand consists of dilation followed by an erosion to eliminate small holes and fill gaps in the image [8]. However, the process of finding optimal threshold value is possibly slower than the previous method since the threshold is calculated differently depending on the amount of noise within an image.

For the 254 (left and right) eye images that were selected by AIQM from distant video in MBGCv1, the previous segmentation rate of pupil boundary in VASIR was $91.9 \%$. Using this new method, the segmentation rate improved to $93.3 \%$. As shown in Figure 4, we found this method to be much more effective for reducing the noise.

The iris boundary is segmented by using a circular Hough transform initially applied by Wildes et al [9]. We use the information of the detected pupil circle as a reference to detect the iris boundary. The eyelid curves are segmented by detecting four points and fitting two ellipses; we will refer to them as upper and lower eyelid points and left and right eyelid corners. The upper and lower eyelid points are detected by a linear Hough transform. For the left and right eyelid corners, our algorithm retrieves all contours that link horizontal segments.

\section{B. Matching}

The Hamming distance (HD) counts the number of positions (in two templates of the same size) for which the binary digits are different [10]. The HD can be used to decide whether or not two iris templates are of the same person. A noise mask [4] helps to exclude the insignificant bits of a template using the equation:

$$
H D=\frac{\sum_{i=1}^{N}\left(t_{i} \oplus q_{i}\right) \cap\left(t m_{i} \cap q m_{i}\right)}{N-\sum_{k=1}^{N}\left(t m_{k} \cup q m_{k}\right)}
$$

where $\mathrm{t}$ and $\mathrm{q}$ are two bit-wise templates and tm and qm are the corresponding noise masks. $\mathrm{N}$ is the total bits of a template. 
For rotational inconsistencies between two iris templates, one template is two bit-wise shifted left or right and the HD value is selected from successive shifts [11].

However, this method may lead to matching failure because the pupil and iris may not be segmented to the exact circle boundaries - especially when the image has significant noise from motion blur or inconsistent lighting conditions. In reality, the iris outer boundary is not uniquelydefined due to its gradual gradient change from the iris to the sclera. Figure 5 illustrates a case of lessthan-optimal segmentation of the pupil (a) and (b) iris boundaries.

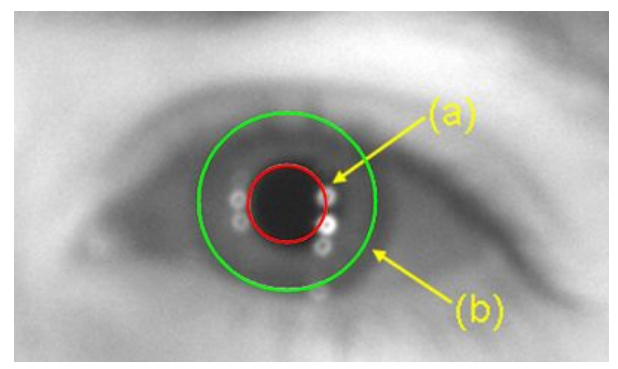

Figure 5 Example of inaccurate segmentation (a) pupil and (b) iris

We therefore propose a new matching method in which the template is shifted not only left and right (horizontal) bit-wise, but also upward and downward (vertical); the Hamming distance values for these $\mathrm{X}$ and $\mathrm{Y}$ shifts are indicated by HD_X and HD_Y, respectively. Figure 6 shows the detailed HD shifting method.

Further, we select successive shift from the values of X or Y (HD_XorY). Finally, the template can also shift in two directions at the same time - HD_XandY. For instance, when one template shifts by one bit to the left, it can also shift one bit up. The new matching method can help to cover both the rotational inconsistency and the segmentation inaccuracy.

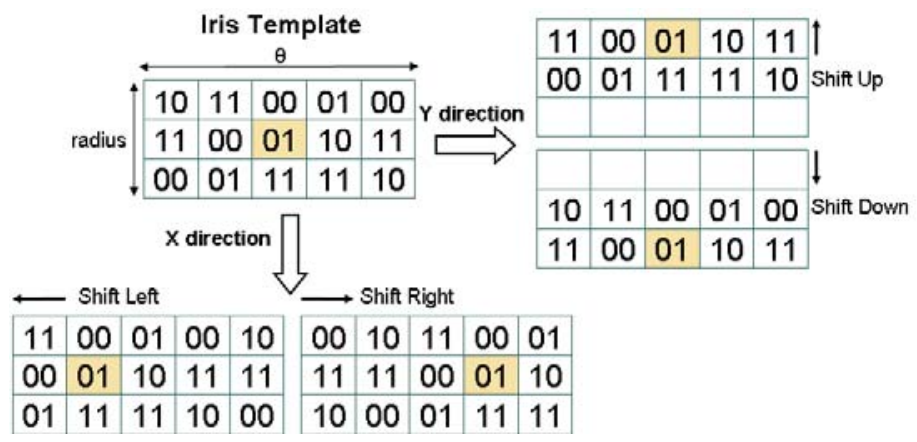

Figure 6 VASIR matching method

For all three matching cases - details are described in section $\mathrm{V}$ - of a) classical-still to classicalstill (SvsS) b) distant-video to classical-still (VvsS), and c) distant-video to distantvideo (VvsV) in Figure 7 illustrates the Hamming distance (HD) comparison of the X, Y, XorY, and XandY matching methods for both the left and right eye based on the MBGCv2 dataset. For SvsS, the matching scores are ordered by XorY, XandY, X, and then Y methods consequence to the results fro $\mathrm{m}$ the ICE2005 dataset. XorY also leads with the best results followed by the Y, X, and XandY for Vv sS. Interestingly, $\mathrm{Y}$ is better for $\mathrm{VvsV}$, (opposite to the results of SvsS) and then XorY, X, XandY foll ow in order. Overall, XorY is better because XorY is best or second best in all three cases. 


\section{Experiments}

In this section, we describe which performance metrics are applied and how three different experiments are designed and executed to evaluate VASIR performance.

Biometric systems are conventionally evaluated by the True Accept Rate (TAR) where two biometric samples are correctly decided to be the same person and the True Reject Rate (TRR) where the system correctly decided that two samples (genuine and imposter) are not the same person. The False Accept Rate (FAR) is defined as the rate where people are accepted as the same identity while in reality they are different and False Reject Rate (FRR) is where two samples from the same person are rejected. The TRR and FRR can be computed as FRR=1-TAR and TRR=1-FAR, respectively. The TAR and FAR are normally obtained by varying an acceptance decision threshold $\lambda$.

The Error Equal Rate (EER) is defined as the rate where $\operatorname{FAR}(\lambda)$ and $\operatorname{FRR}(\lambda)$ are the same value (i.e., intersect on a FAR and FRR vs. $\lambda$ plot). The Detection Error Trade-off (DET) curve is a graph of FAR against FRR. The Receiver Operator Characteristic (ROC) curve is a plot of TAR against FAR. The DET and ROC curves are useful, but, neither of these plots provides the detailed threshold (e.g., HD) information needed to estimate the system performance. We therefore add two additional curves to evaluate VASIR performance: False Curves with Threshold distribution (FCT), and True Curves with Threshold distribution (TCT). FCT is the plot of FAR and FRR against $\lambda$; whereas TCT is the opposite; TAR and TRR against $\lambda$. These curves allow us to analyze FAR and FRR with EER and to represent the discrepancy between matching and non-matching distribution at the same time.

In terms of Verification (1:1), an image matches if its matching score has satisfied a certain threshold $\lambda$, whereas Identification (1:Many) indicates that an image matches if its score is the best. Our results are based on verification.

\section{A. Comparison of VASIR and IrisBEE}

Is VASIR robustly superior to IrisBEE? In order to answer this question, the ICE 2005 evaluation protocol [2] was applied to measure VASIR's performance for comparison purposes. An algorithm compares a query image qi to a target image ti to produce the similarity scores for matching and nonmatching. As mentioned in section four, only classical-still to classical-still matching is allowed for this dataset. It is of note that the images for the subjects were captured on different days.

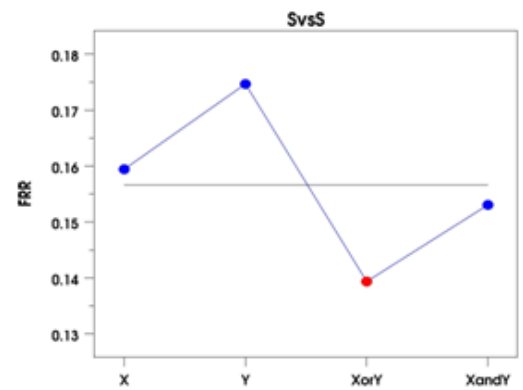

(a)

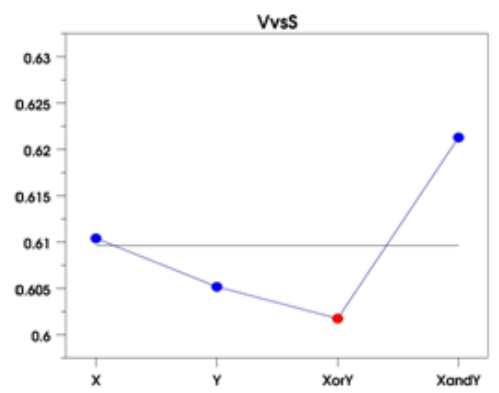

(b)

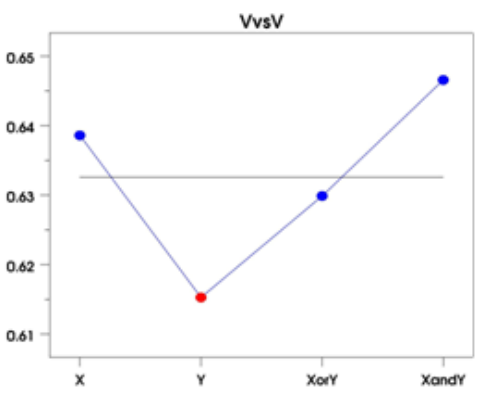

(c)

Figure 7 Comparison of four matching methods for each of three data types

(a) classical-still to classical-still (SvsS) (b) distant-video to classical-still (VvsS)

(c) distant-video to distant-video (VvsV) 
The four types of matching methods (HD_X, HD_Y, HD_XorY and HD_XandY) - mentioned in section 3 - were evaluated with two criteria for the comparison; 1) TAR at FAR of 0.001 (for which higher is better) and 2) the EER value (for which smaller is deemed as better).

The performance results from VASIR's HD_XorY has the highest TAR at FAR of 0.001 and the smallest EER both for the left and right dataset in ICE2005; HD_XandY, HD_X and HD_Y follow in order. Figure 8 shows the comparison between IrisBee's and VASIR's results by plotting the ROC curve. The plot visualizes IrisBEE (HD_X) and VASIR (HD_XorY).

For the left eye, at FAR of 0.001 , VASIR has a TAR of $88.5 \%$ while IrisBEE is only $85.0 \%$. For the right eye, VASIR's TAR is $90.3 \%$ while IrisBEE's TAR is $85.2 \%$. Hence for both eyes, VASIR is an improvement over IrisBEE. For the EER criterion - (not shown), VASIR's EER is better with $\sim 4.4 \%$, while IrisBEE's EER is nearly twice larger.
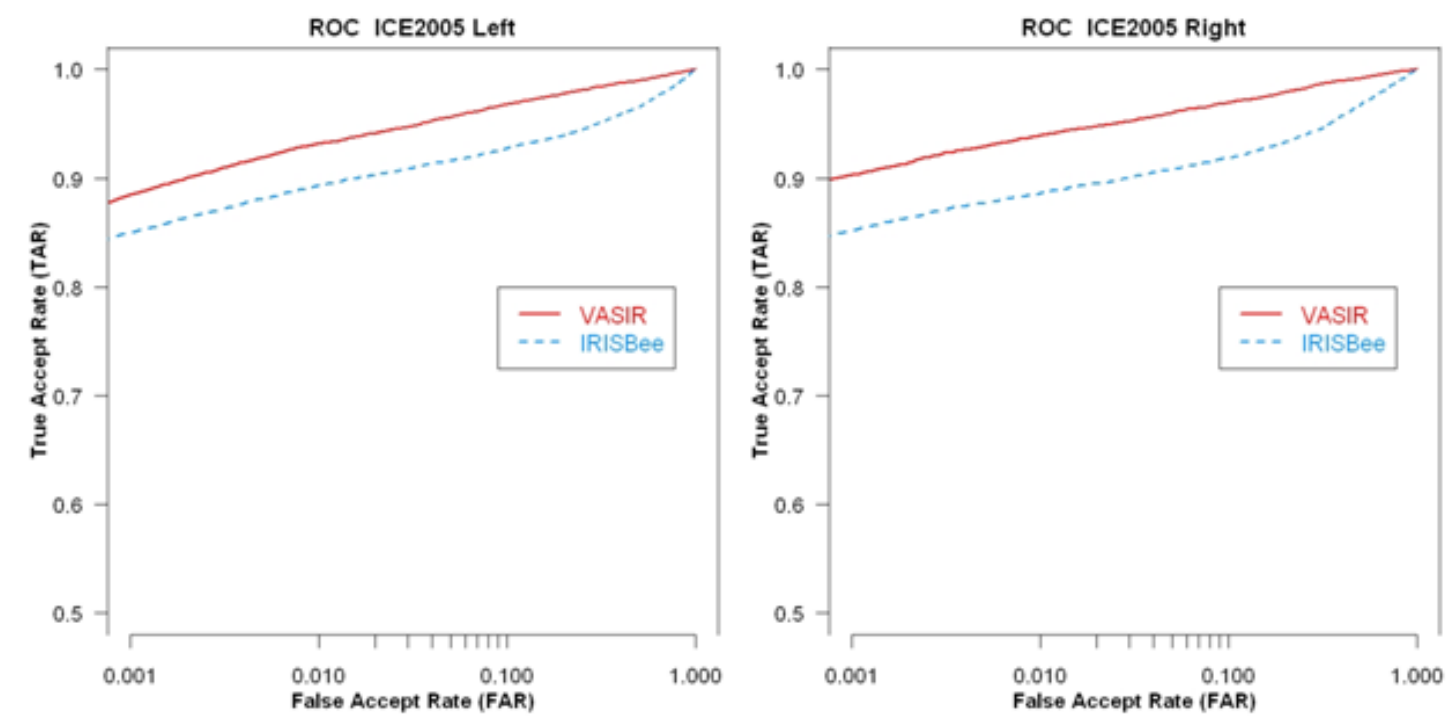

Figure 8 Comparison of VASIR and IrisBEE for the left and right eye (classical-still to classical-still iris images matching)

The results show that even though VASIR was developed primarily as a tool for distant-videobased iris recognition, the system is robust for classical-still-based iris recognition as well.

\section{B. Baseline for Benchmarking}

Can VASIR's results be used as a state-of-the-art baseline for both classical-still-based and distant-video-based iris recognition? We present the performance results based on the MBGCv2 dataset using VASIR for the purpose of providing a baseline both under unconstrained distant-videobased and constrained classical-still-based iris recognition. They can be used as a reference for subsequent evaluation or for comparison with custom algorithms. These results may also provide an opportunity for other researchers to advance their iris recognition algorithms and to promote their technology.

For distant-video data, the eye region was detected in 598 videos out of the total 628 videos. In 30 videos that were included either no eyes were visible in the video or the file was erroneous. The number of extracted iris images for the left and right eye was 9,592 out of the total 11,341 frames. No 
eye region could be detected in 41 images - out of the total 9,592 images (false detection rate: $0.43 \%$ ). For classical-still dataset, 4,025 images for the left and 4,013 images for the right eye were evaluated.

Furthermore, we provide a prototype of our experiment design showing how to evaluate performan ce results.

(a)

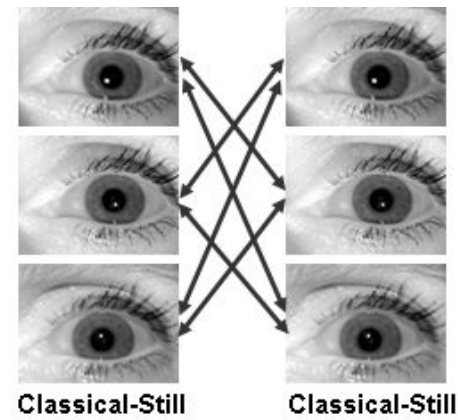

(b)

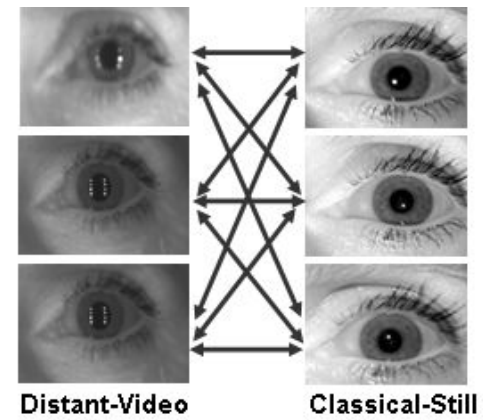

(c)

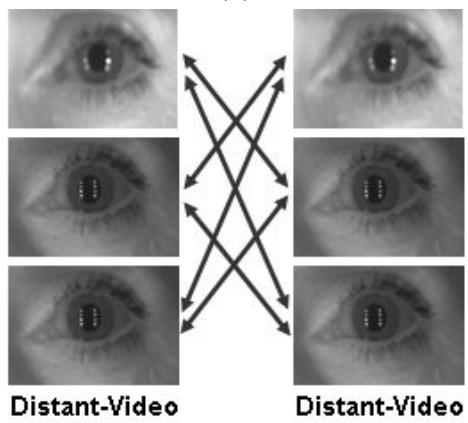

Figure 9 Three matching protocol

(c) distant-video to distant-video (VvsV)

First, VASIR allows us to match 1) classical-still to classical-still (SvsS) 2) distantvideo to classical-still (VvsS), and 3) distant-video to distant-video (VvsV). For SvsS and VvsV (allframe to all-frame) matching scores, the query image qi matches the target images ti where $i$ is the sa me person, but a different image regardless of the captured date of video sequences or still images. In VvsS matching scores, the query matches all targets where the subject is the same person. An advanta ge of this protocol is that the user does not have to repeatedly generate the mask for each dataset, the $\mathrm{s}$ ystem is using the subject ID which is part of the file name. Figure 9 shows how matching scores were calculated for each procedure.

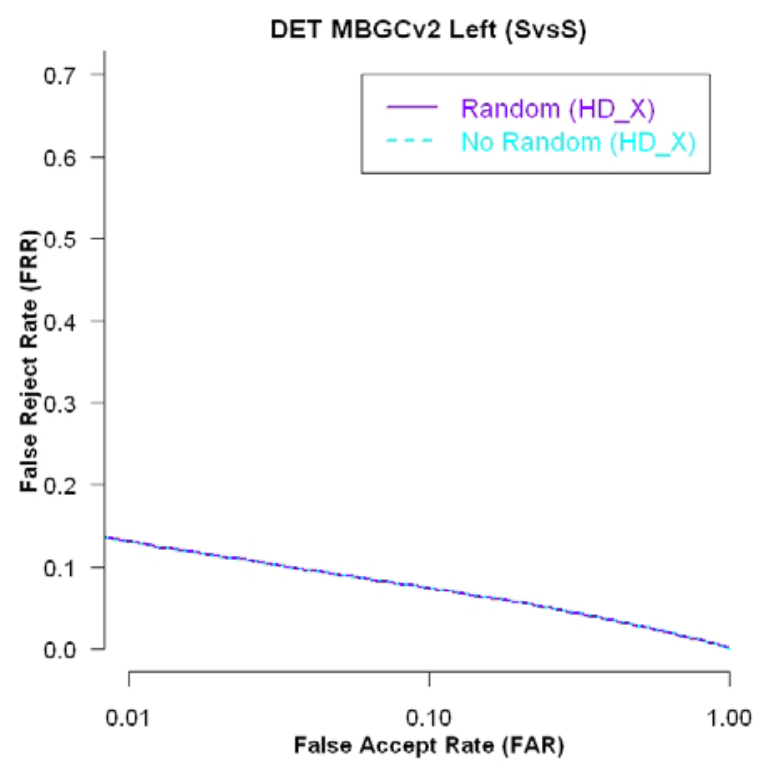

Figure 10 Comparison of full non-matching $(1,638,949$ entries) and random non-matching (201,250 entries) out of 4,025 NIR_Iris_Still images;

There are at least 6 images per person per eye 
For non-matching scores, the query qi compares against all targets $t i$ where the target subject is a different person. This full matching (one to all others) can be time-consuming and is not necessary since the non-matching scores are sufficiently consistent for virtually all query subjects. We therefore suggest randomly selecting a subsample of targets, all of a different person for getting the nonmatching results; we found 50 targets to be a sufficient subsample. This not only reduces time cost but also allows us to characterize the performance for analysis purposes more easily. Figure 10 provides the DET curve obtained from all left eye images on MBGCv2 SvsS non-matching. The results show that in comparison full non-matching and random non-matching are nearly equivalent.

In Figure 11 (four plots of the left and right ROC and FCT curves), we compare the performance $r$ esults of SvsS, VvsS, and VvsV matching using HD_XorY matching method based on the results as $\mathrm{s}$ hown in Figure 7. The detailed error rates are described in Table 4. The results show that SvsS matchi ng has the best performance; VvsS and VvsV follow next as expected.

Table 4 VASIR's Performance results

\begin{tabular}{|c|c|c|c|}
\hline \multirow{2}{*}{ Datasets } & \multicolumn{2}{|c|}{ TAR at FAR $=0.01$} & \multirow{2}{*}{ EER } \\
\cline { 2 - 3 } & LEFT & RIGHT & \\
\hline SvsS & $89.5 \%$ & $89.8 \%$ & $6.7 \%$ \\
\hline VvsS & $25.3 \%$ & $33.5 \%$ & $30.9 \%$ \\
\hline VvsV & $24.5 \%$ & $33.1 \%$ & $32.6 \%$ \\
\hline
\end{tabular}
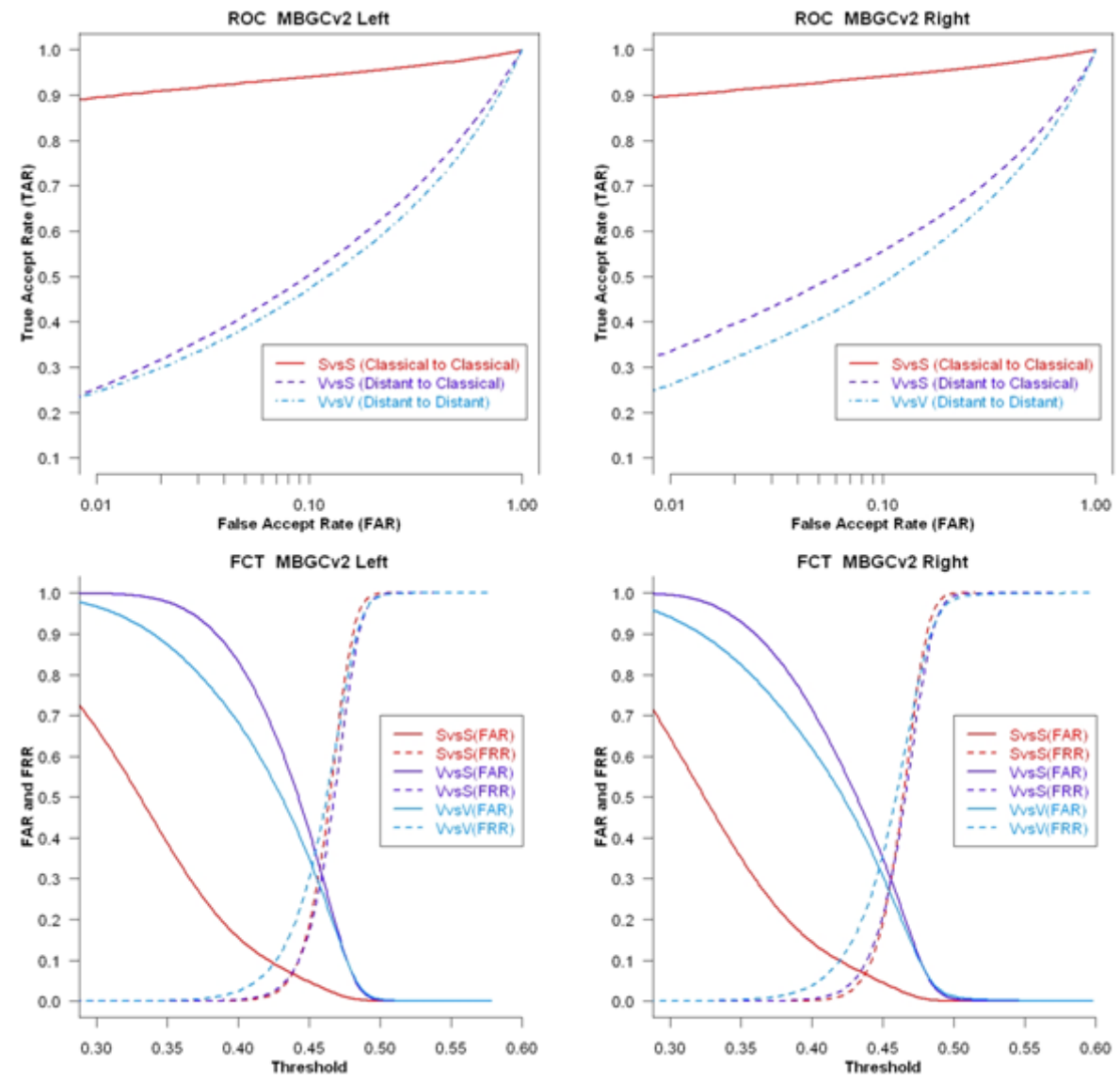

Figure 11 Comparison of SvsS, VvsS, and VvsV for the left and right 


\section{Image Quality Scores and Performance}

How does VASIR performance change with image quality? Although several metrics[13][14] were used to address one or another aspect of image quality metrics, the edge density - as defined by the Sobel operator - was our primary image quality metric of choices. After first using the edge density metric to determine focus, the metric was again applied to arrive at a final order categorization of the quality of specific images.

The Sobel operator is designed to respond to edges running vertically and horizontally relative to $t$ he pixel grid. The $D x$ and $D y$ are applied separately to the input image to measure the gradient compo nent in each orientation. The gradient magnitude, which a higher score is considered better image qual ity in our evaluation, can be given by:

$$
Q=\sqrt{D x^{2}+D y^{2}}
$$

The quality score from Flynn et al [15] is measured for a pair of samples $q$ and $t$ and is defined as,

$$
Q(q, t)=\min (Q(q), Q(t))
$$

To calculate a quality measure $Q(q, t)$ for a set of match scores $M$ and a set of nonmatch scores $N$, decision threshold $\lambda$ and image quality threshold $\tau$ are given as parameters. Therefor $\mathrm{e}, \operatorname{TAR}(\lambda, \tau)$ and $\operatorname{FAR}(\lambda, \tau)$ are computed as defined,

$$
\operatorname{TAR}(\lambda, \tau)=\frac{\#\{M \cap S(q, t) \geq \lambda \cap Q(q, t) \geq \tau\}}{\#\{M \cap Q(q, t) \geq \tau\}}
$$

$M$ is the set of match similarity scores and $\mathrm{S}(\mathrm{q}, \mathrm{t})$ is the similarity score where the two images $q$ and $t$ are of the same person. The TAR is the fraction of similarity score $S(q, t)$ greater than or equal to a de cision threshold $\lambda$, where the quality score $Q(q, t)$ is greater than or equal to a threshold $\tau$.

$$
\operatorname{FAR}(\lambda, \tau)=\frac{\#\{N \cap S(q, t) \geq \lambda \cap Q(q, t) \geq \tau\}}{\#\{N \cap Q(q, t) \geq \tau\}}
$$

$N$ is the set of non-match similarity scores and $S(q, t)$ is the similarity score where the two images $q$ and $t$ are of the different persons. The FAR is the fraction of similarity score $\mathrm{S}(\mathrm{q}, \mathrm{t})$ greater than or equal to a decision threshold $\lambda$, where the quality score $Q(q, t)$ is greater than or equal to a threshold $\tau$. 


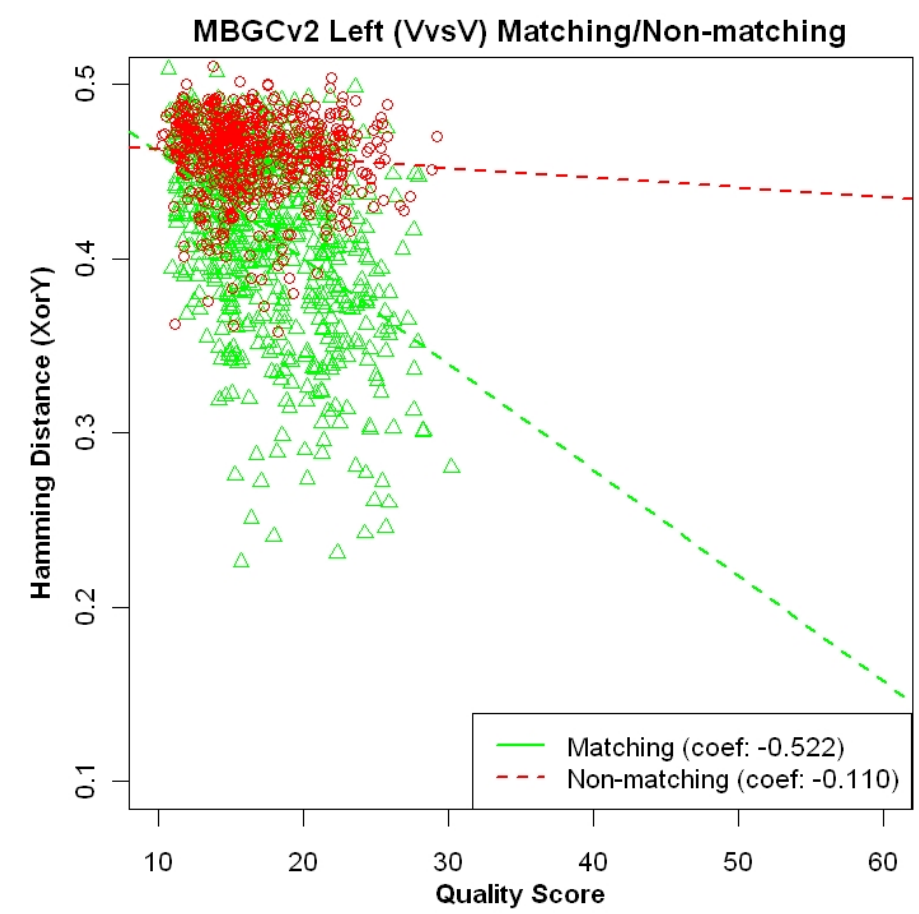

Figure 12 Distribution of quality score (edge desnity) and Hamming distance (XorY) on VvsV matching and non-matching

Figure 12 shows the distribution of the quality scores and Hamming distances (XorY) based on left and right images for distant-video to distant-video (VvsV). The plot illiustrates that the VvsV dataset consists of poor quality images; the scatter points are primarily found on the left side. The Hamming distance of non-matching scores is mainly found in the top quarter of the plot. The results show that VASIR's performance and image quality scores (edge density) are correlated with the coefficient value -0.52 on $\mathrm{VvsV}$ matching, while the non-matching has only -0.11 . Figure 13 depicts actual examples of $\mathrm{VvsV}$ highest and lowest quality score.

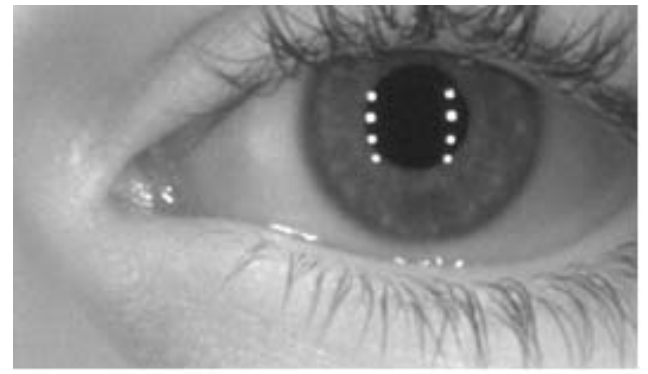

(a)

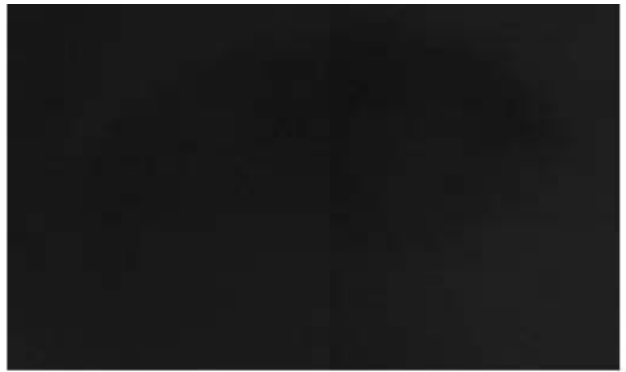

(b)

Figure 13 Samples ordered by quality score (edge density)

(a) VvsV: Highest score image (341x200px) (b) VvsV: Lowest score image (506x301px) 

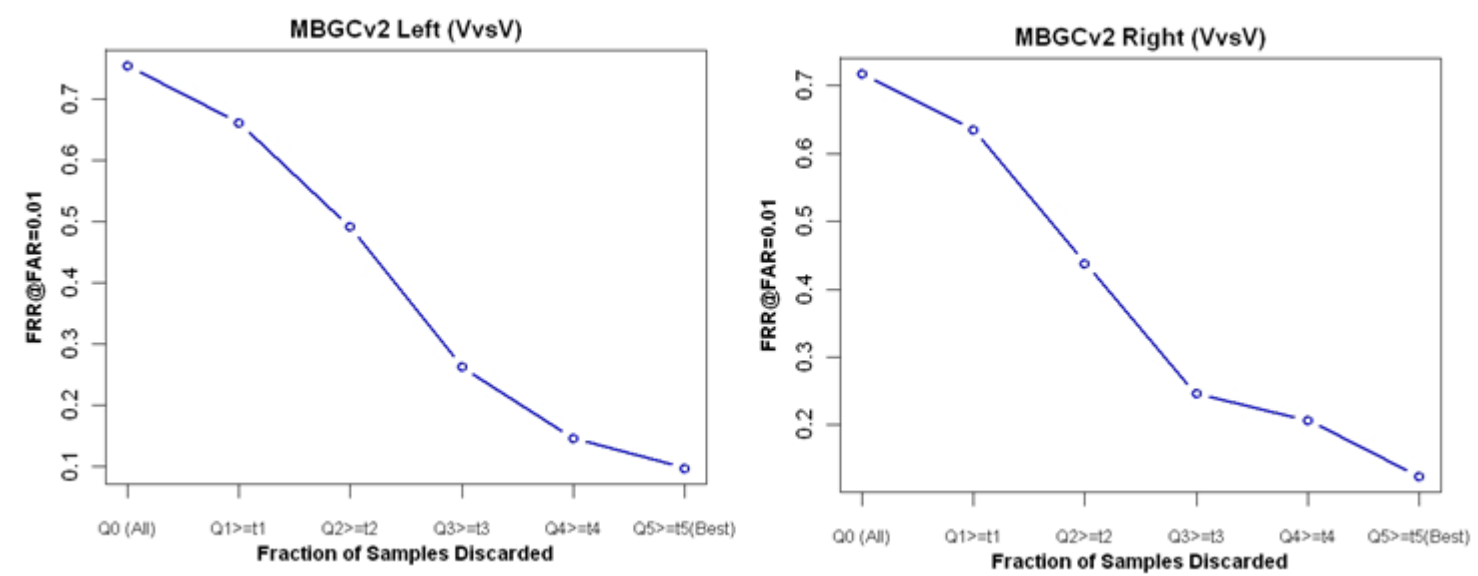

Figure 14 VASIR's performance on the image quality for distant-video to distant-video matching

Following, the False Reject Rate (FRR) is calculated by applying six levels of the image quality. In case of the left eye images, the quality score for $\mathrm{VvsV}$ is calculated with the edge density ranges approximately from 10 to 35 . We define a set of six quality levels ( 0 to 5 ) with thresholds $\left\{\tau_{0} \ldots \tau_{5}\right\}$;

Quality level $0(\mathrm{Q} 0)$ is all images; Quality level $1(\mathrm{Q} 1)$ means all images with quality score $\geq \tau_{1}$; the remaining levels are $\mathrm{Q} 2 \geq \tau_{2}, \mathrm{Q} 3 \geq \tau_{3}, \mathrm{Q} 4 \geq \tau_{4}$, and $\mathrm{Q} 5 \geq \tau_{5}$ (best).

For each quality level, VASIR's performance is evaluated with an FRR value of FAR at 0.01, using only samples of all quality levels above the current level. Figure 14 illustrates the VvsV performance results of the eye images with the matching method by applying HD_XorY.

The plots in Figure 14 show that the FRR in VvsV performance is reduced for both left and right when low quality images are_excluded. It is important to note that the image quality was measured from the datasets "as-is" without localizing the iris region in an image. The possible problem is that the quality score will be high if the image includes a lot of noise such as eyebrows and eyelashes.

The positive side of these results is that the image quality (as measured via the edge density) may serve as a predictor of VASIR performance on VvsV matching. However, there is a need for analyzing quality metrics for good image quality datasets such as classical-still iris images. We are pursuing the investigation of this matter as well. 


\section{Conclusion}

VASIR was evaluated using the ICE 2005 and MBGC datasets. Using the ICE 2005 dataset, at FAR of 0.001 , VASIR has a TAR of $88.5 \%$ while IrisBEE is only $85.0 \%$ for the left eye. VASIR's TAR is $90.3 \%$ while IrisBEE's TAR is $85.2 \%$ for the right eye. For the EER criterion (where small is better), VASIR is $\sim 4.4 \%$ for the left and right eyes while IrisBEE's EER is considerably worse at twice the value. The results show that VASIR is superior to IrisBEE.

The four matching methods in VASIR were evaluated using the MBGC dataset: For classical-still to classical-still (SvsS), the matching HD scores are ordered by XorY, XandY, X, and then Y. For distant-video to classical-still (VvsS), XorY also leads with results superior to Y, X, followed by XandY. Interestingly, for distant-video to distant-video (VvsV), $\mathrm{Y}$ is better (opposite to the results of SvsS) and then XorY, X, XandY in that order. Overall, XorY is better because XorY is best or second best in all three cases.

We also provided a baseline of performance results on SvsS, VvsS, and VvsV iris recognition under a broad range of image and environmental conditions.

Finally, our study shows that image quality is correlated with VASIR performance on VvsV matching. Hence, the quality score metrics may be served as a predictor of VASIR performance.

Various quality metrics on VASIR performance are currently in progress. Upon approval of manag ement, we plan to release the VASIR algorithm.

\section{ACKNOWLEDGMENT}

The identification of any commercial product or trade name does not imply endorsement or recommendation by the National Institute of Standards and Technology.

\section{REFERENCES}

[1] Y. Lee, P. J. Phillips, R. J. Micheals, "An Automated Video-Based System for Iris Recognition", Proc. of The 3rd IAPR/IEEE International Conference on Biometrics, LNCS 5558, pp.1167-1176, 2009.

[2] P. J. Phillips, K. W. Bowyer, P. J. Flynn, X. Liu, andW. T. Scruggs, "The iris challenge evaluation 2005," in Proc. IEEE Int. Conf. on Biometrics: Theory, Applications, and Systems (BTAS), 2008.

[3] P. J. Phillips, T. Scruggs, P. J. Flynn, K.W. Bowyer, R. Beveridge, G. Givens, B. Draper, and A. O'Toole, "Overview of the multiple biometric grand challenge," in Proc. Int. Conf. on Biometrics (ICB), 2009.

[4] L. Masek, Recognition of Human Iris Patterns for Biometric Identification, The University of Western Australia, http://www.csse.uwa.edu.au/ pk/studentprojects/libor/

[5] J. G. Daugman, M.S: High Confidence Visual Recognition of Persons by a Test of statistical Independence, IEEE Transactions on Pattern Analysis and Machin Intelligence, VOL, 15, NO. 11,1993 
[6] P. Yao, J. Li, X. Ye, Z. Zhuang, and B. Li., C.: Iris Recognition Algorithm using Modified Log-Gabor Filters, Proceedings of International Conference on Pattern Recognition, pages 461-464, 2006.

[7] Y. Lee, R. J. Micheals, P. J. Phillips, "Improvements in video-based automated system for iris recognition (VASIR). In Motion and Video Computing, 2009. WMVC '09. Workshop on, pages 1--8, 2009

[8] R. C. Gonzalez and R. E. Woods. Digital Image Processing. Addison-Wesley Publishing Company, 1992.

[9] R. P. Wildes, "Iris Recogniton: An Emerging Biometric Technology", Proceedings of the IEEE, VOL.85, No. 9, 1997.

[10] R. W. Hamming, "Error detecting and error correcting codes", Bell System Technical Journal 29 (2): 147-160, MR0035935, 1950.

[11] J. Daugman, C.: How iris recognition works, Proceedings of International Conference on Image Processing, Vol. 1, 2002.

[12] J. R. Matey and O. Naroditsky and K. Hanna and R. Kolcynksi and D. J. LoIancono and S. Mangru and M. Tinker and T. M. Zappa and W. Y. Zhao, "Iris on the Move: Acquisition of Images for Iris Recognition in Less Constrained Environments" in proceedings of the IEEE, Vol. 94, No. 11, 2006.

[13] N.Kalka, V.Dorairaj, Y.Shah, N.A.Schmid, and B.Cukic, "Image Qaulity Assessment for Iris Biometric", in proceedings of the Research Session, Biometrics Consortium Conf.pp.58-59, 2005.

[14] J Ross Beveridge, Geof H Givens, P Jonathon Phillips, Bruce A Draper, Yui Man Lui, "Focus on Quality, Predicting FRVT 2006 Performance", 8th IEEE International Conference on Automatci Face and Gesture Recogniton (FG), 2008.

[15]P. J. Flynn, P.J. Phillips, "ICE Mining: Quality and Demographic Investigations of ICE 2006 Performance Results", Multiple Biometrics Grand Challenge (MBGC) Kick-Off Workshop, 2008. 